\title{
Prognostic role of baseline neutrophil-to-lymphocyte ratio in metastatic solid tumors
}

\author{
AMANDA ZUCKER $^{1 *}$, ALEX WINTER $^{1 *}$, DEAN LUMLEY $^{1}$, \\ PAWEL KARWOWSKI ${ }^{2}$, MIN-KYUNG JUNG ${ }^{1}$ and JOHNNY KAO ${ }^{2}$ \\ ${ }^{1}$ College of Osteopathic Medicine, New York Institute of Technology, Glen Head, NY 11545; \\ ${ }^{2}$ Department of Radiation Oncology, Good Samaritan Hospital Medical Center, West Islip, NY 11795, USA
}

Received March 14, 2019; Accepted June 15, 2020

DOI: $10.3892 / \mathrm{mco} .2020 .2095$

\begin{abstract}
High baseline neutrophil-to-lymphocyte ratio (NLR) has been associated with poor survival in a number of solid tumors, but has not been extensively investigated in the context of radiation oncology. Developing more robust models to predict survival would inform patient care for patients with metastatic solid tumors. The present study was undertaken to evaluate the effect of baseline NLR (using 4 as a cutoff) on survival in 320 consecutive patients with metastatic cancer who were referred to a single radiation oncologist between 2012 and 2015, with a median follow-up of 20.6 months. The median NLR was 4.4 (interquartile range, 2.8-7.2). Patients with a baseline NLR $\leq 4$ had a median survival of 9.3 months compared to 4.1 months for NLR $>4(\mathrm{P}<0.001)$. The number of active tumors, Eastern Cooperative Oncology Group performance status score, baseline albumin, primary tumor site, liver metastases and baseline NLR predicted overall survival on both univariate and multivariate analysis $(\mathrm{P}<0.05$ for all). After adjusting for known prognostic factors for advanced solid tumors, baseline NLR $>4$ independently predicted adverse survival in this cohort.
\end{abstract}

\section{Introduction}

Tumor-promoting inflammation by innate immune cells fosters multiple hallmarks of cancer (1). The neutrophil-to-lymphocyte ratio (NLR) is a widely utilized marker of host inflammation (2). High baseline NLR (>4) has been demonstrated to have prognostic significance for overall survival in solid tumors, with a hazard ratio of 1.8 (3). Our group has previously

Correspondence to: Dr Johnny Kao, Department of Radiation Oncology, Good Samaritan Hospital Medical Center, 1000 Montauk Highway, West Islip, NY 11795, USA

E-mail: johnny.kao@chsli.org

*Contributed equally

Key words: neutrophil-to-lymphocyte ratio, radiation, survival prediction validated the NEAT model, including the number of active tumors ('N'), Eastern Cooperative Oncology Group (ECOG) performance status ('E'), albumin ('A'), and primary tumor site ('T') as important prognostic factors for patients with metastatic cancer referred to radiation oncology $(4,5)$. Other validated models identified age, extent of prior chemotherapy, recent hospitalization, liver metastases and bone-only metastases as prognostic factors for radiation oncology patients with metastatic cancer $(6,7)$. However, despite extensive research, these validated models only accounted for 30-50\% of observed survival (5).

The prognostic role of baseline NLR has been demonstrated in 100 studies for several types of solid tumors (3). To date, limited data has been provided for NLR in patients treated with radiation, particularly in those with metastatic solid tumors $(8,9)$. Previous studies have demonstrated that patients with metastatic disease treated with radiation tend to have a baseline NLR of 4-5 $(8,9)$. By contrast, the baseline NLR for the general population is $1.65-2.15(10,11)$. Since NLR is a widely available and cost-effective measure of systemic inflammation, the current study hypothesized that elevated NLR may add further prognostic value, thereby improving predictive accuracy.

\section{Materials and methods}

Inclusion criteria. The present retrospective study included 320 consecutive patients (age range, 23-97 years), with metastatic stage IV solid tumor who were referred to a single physician in a large community hospital-based radiation Oncology Department between May 2012 and October 2015. This minimal risk study was approved by the Good Samaritan Hospital Medical Center Institutional Review Board.

Data collection. The charts and electronic records of all patients were assessed for previously validated prognostic factors, including ECOG performance status score (ECOG 0-1 vs. ECOG 2 vs. ECOG 3-4), primary tumor type (breast, prostate, kidney, lung or other), number of tumors (1-5 vs. $\geq 6$ ), serum albumin ( $\geq 3.4$ vs. $2.4-3.3$ vs. $\leq 2.4 \mathrm{~g} / \mathrm{dl}$ ), location of metastases (bone only vs. liver vs. other) and age ( $>60$ years vs. $\leq 60$ years). Additionally, the relative contribution of baseline NLR (>4 vs. $\leq 4)$ at the time of consultation was explored. 
Table I. Predictors of overall survival.

\begin{tabular}{|c|c|c|c|c|}
\hline Variables & Number (\%) & P-value & Median survival (months) & 6 -month survival (\%) \\
\hline NLR & & $<0.001$ & & \\
\hline$\leq 4$ & $129(53)$ & & 9.3 & 64.6 \\
\hline$>4$ & $169(40)$ & & 4.1 & 41.4 \\
\hline Unknown & $22(7)$ & & & \\
\hline ECOG performance status score & & $<0.001$ & & \\
\hline $0-1$ & $125(39)$ & & 16.4 & 81.4 \\
\hline 2 & $93(29)$ & & 5.9 & 48.0 \\
\hline $3-4$ & $102(32)$ & & 1.7 & 20.9 \\
\hline Primary tumor & & $<0.001$ & & \\
\hline Breast, prostate or kidney & $83(26)$ & & 14.3 & 76.3 \\
\hline Other & $237(74)$ & & 5.2 & 45.3 \\
\hline Number of active tumors & & $<0.001$ & & \\
\hline$\leq 5$ & $87(27)$ & & 18.3 & 80.8 \\
\hline$>5$ & $233(73)$ & & 4.8 & 43.1 \\
\hline Albumin (g/dl) & & $<0.001$ & & \\
\hline$>3.3$ & $155(52)$ & & 12.7 & 69.9 \\
\hline $2.4-3.3$ & $114(38)$ & & 3.8 & 33.2 \\
\hline$<2.4$ & $30(10)$ & & 1.5 & 20.8 \\
\hline Unknown & $21(7)$ & & & \\
\hline Age (years) & & 0.06 & & \\
\hline$>60$ & $81(25)$ & & 6.1 & 51.6 \\
\hline$\leq 60$ & $239(75)$ & & 9.5 & 58.4 \\
\hline Liver metastases & & $<0.001$ & & \\
\hline No & $254(79)$ & & 7.9 & 58.2 \\
\hline Yes & $66(21)$ & & 4.0 & 35.1 \\
\hline Bone-only metastases & & $<0.001$ & & \\
\hline No & $270(84)$ & & 5.9 & 49.0 \\
\hline Yes & $50(16)$ & & 15.5 & 77.2 \\
\hline Recent hospitalization & & $<0.001$ & & \\
\hline No & $113(35)$ & & 13.8 & 75.8 \\
\hline Yes & $207(65)$ & & 4.1 & 40.6 \\
\hline Lines of palliative chemotherapy $\geq 2$ & & 0.75 & & \\
\hline No & $281(88)$ & & 6.7 & 53.4 \\
\hline Yes & $39(12)$ & & 6.2 & 53.4 \\
\hline
\end{tabular}

NLR, neutrophil-to-lymphocyte ratio; ECOG, Eastern Cooperative Oncology Group.

Statistical analysis. Statistical analysis was performed with Stata 13.1 (StataCorp LP). The primary outcome was overall survival, defined as the time from the initial radiation oncology consultation to the date of death from any cause. Patients were included regardless of treatment received, and patients who were lost to follow-up were censored at the date of the last follow-up. Survival data were analyzed using the Kaplan-Meier method and summarized by median and 6-month survival. Differences in survival were assessed through the log-rank method. The association of individual variables with survival was assessed using the Cox proportional hazards model that was verified by tests of correlations over time with examination of residual plots. The Pearson $\chi^{2}$ test was performed to determine whether there were baseline differences in variables that were associated with high or low NLR. Statistical tests were two-sided, and $\mathrm{P}<0.05$ was considered to indicate a statistically significant difference.

Patient characteristics. The study population included 320 patients with distant metastases who were referred for radiation oncology evaluation, with $55 \%$ inpatient and $45 \%$ outpatient consultations. The median age was 68 years (interquartile range, 60-78 years), the median NLR was 4.4 (interquartile range, 2.8-7.2) and the median albumin was $3.4 \mathrm{~g} / \mathrm{dl}$ (interquartile range, 2.8-3.8 g/dl). In terms of performance status, $39 \%$ of the patients were ECOG $0-1,29 \%$ were 
Table II. Cox multivariable analysis.

\begin{tabular}{lccc}
\hline Variables & Hazard ratio & 95\% confidence interval & P-value \\
\hline Primary tumor site (breast, kidney, or prostate vs. other) & 3.30 & $2.24-4.86$ & $<0.001$ \\
Number of active tumors (1-5 vs. $\geq 6$ ) & 2.90 & $2.04-4.11$ & $<0.001$ \\
ECOG performance status score (0-1 vs. 2 vs. 3-4) & 2.24 & $1.85-2.73$ & $<0.001$ \\
Serum albumin ( $\geq 3.4$ vs. $2.4-3.3$ vs. $<2.4$ g/dl) & 1.52 & $1.22-1.90$ & $1.09-2.11$ \\
Liver metastasis (no vs. yes) & 1.52 & $1.01-1.78$ & 0.01 \\
NLR ( $\leq 4$ vs. $>4$ ) & 1.34 & $0.78-1.54$ & 0.04 \\
Prior hospitalizations within the last 3 months (0 vs. $\geq 1)$ & 1.10 & $0.62-1.45$ & 0.59 \\
Metastasis location (bone only vs. other) & 0.94 & $0.65-1.48$ & 0.79 \\
No. of prior palliative chemotherapy courses (0-1 vs. $\geq 2)$ & 0.98 & $0.81-1.23$ & 0.93 \\
Age ( $\leq 60$ years vs. $>60$ years) & 1.00 & 0.98
\end{tabular}

NLR, neutrophil-to-lymphocyte ratio; ECOG, Eastern Cooperative Oncology Group.

ECOG 2 and 32\% were ECOG 3-4. The most common types of cancer included non-small cell lung cancer (34\%), breast (14\%), small cell lung cancer $(10 \%)$, prostate $(9 \%)$, colorectal $(6 \%)$, kidney (4\%), endometrial (4\%) and unknown primary (4\%). With respect to the extent of the disease, $7 \%$ of the patients had only 1 site of active disease, $7 \%$ had 2 active tumors and $13 \%$ had 3-5 active tumors. Liver metastases were present in $21 \%$ of the patients and $16 \%$ had bone-only metastases. Only $12 \%$ of the patients had previously received $\geq 2$ lines of palliative chemotherapy.

Radiation therapy and systemic therapy. Among the 320 patients evaluated, $81 \%$ were treated with radiation therapy. Treatment was personalized to account for disease site and clinical status and goals of treatment. To summarize, the majority of the patients were treated with standard palliative regimens, most commonly 30 Gy in 10 fractions. Patients with a poor prognosis were either not treated or treated with short-course radiation (8-20 Gy in 1-5 fractions). Patients with limited brain metastatic disease (typically 1 to 4 brain metastases) were often treated with fractionated stereotactic radiotherapy (27 Gy in 3 fractions) or single-fraction stereotactic radiosurgery (21-24 Gy in 1 fraction). Patients with good performance status and oligometastatic disease (defined as $\leq 5$ metastases) were treated with curative intent dose-intensive treatment regimens $(12,13)$.

Systemic therapy was administered at the discretion of the treating medical oncologist and, depending on the diagnosis, consisted of chemotherapy, hormonal therapy, biologically targeted therapy, immunotherapy and/or supportive care.

\section{Results}

Predictors of survival on univariate analysis. The median survival for all patients was 6.7 months, with a median follow-up for surviving patients of 20.6 months. On univariate analysis, baseline NLR, number of tumors, ECOG performance status, serum albumin, primary tumor site, prior hospitalization within the last 3 months, liver metastases, bone-only and liver metastases, were found to be significant predictors of

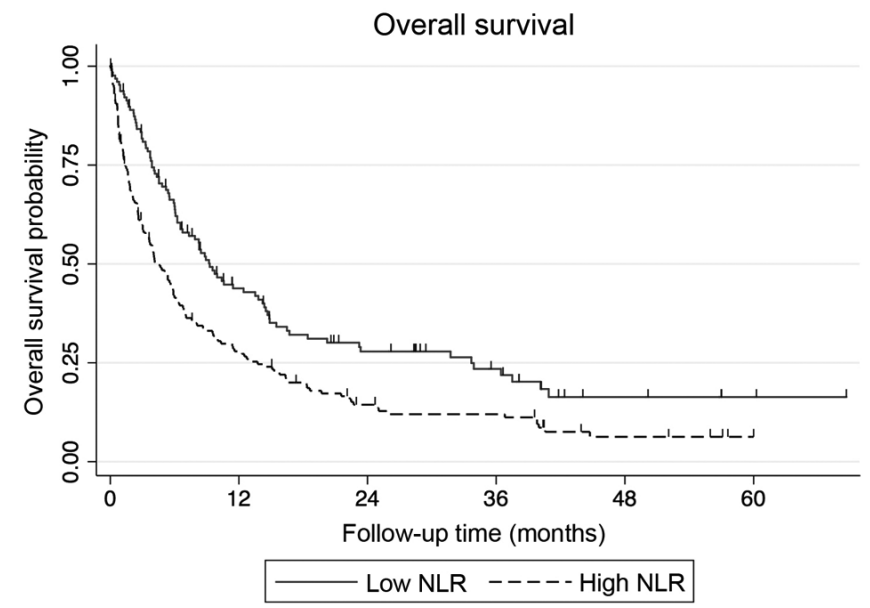

Figure 1. Effect of NLR on the overall survival of patients with metastatic solid tumors. NLR, neutrophil-to-lymphocyte ratio.

survival (Table I). The median survival for patients with an NLR of $\leq 4$ was 9.3 months vs. 4.1 months for those with an NLR of $>4$ (Fig. 1). Age and number of prior palliative chemotherapy cycles were not found to be statistically significant in predicting survival.

Multivariate analysis. On Cox regression analysis, the statistically significant predictors of survival included ECOG performance status $(\mathrm{P}<0.001)$, number of tumors $(\mathrm{P}<0.001)$, primary tumor site $(\mathrm{P}<0.001)$, albumin $(\mathrm{P}<0.001)$, liver metastases $(\mathrm{P}=0.013)$ and baseline NLR $(\mathrm{P}=0.042)$. Age $(\mathrm{P}=0.98)$, number of prior chemotherapy cycles $(\mathrm{P}=0.93)$, recent hospitalization $(\mathrm{P}=0.59)$ and bone-only metastases $(\mathrm{P}=0.79)$ were not found to be statistically significant in predicting survival (Table II).

\section{Discussion}

The present study demonstrated that baseline NLR is an independent predictor of survival in patients with metastatic cancer, even when accounting for potential confounding 
variables, including performance status, albumin, tumor type and extent of the disease. While statistically significant, the $\mathrm{P}$-value and hazard ratio $(\mathrm{P}=0.042$; hazard ratio, 1.34) indicated that only small improvements were observed when compared with the previously published and validated NEAT model, defined as the number of active tumors ('N'), Eastern Cooperative Oncology Group (ECOG) performance status ('E'), albumin ('A'), and primary tumor site ('T') (5). In terms of a predictive model, adding NLR did not significantly improve the performance of the NEAT model, having little effect on the $\mathrm{C}$-index or modified $\mathrm{r} 2$ coefficient of determination (unpublished data).

The mechanism underlying the association of high baseline NLR with poor survival remains elusive. The impact of host antitumor immunity on patient clinical outcome with advanced solid tumors is of great interest to practicing oncologists. Tumors accompanied by significant systemic inflammation behave more aggressively, which likely reflects the contribution of the microenvironment to disease progression (14). Neutrophils are not only antagonists of microbial infection and facilitators of wound healing, but also promoters of cancer initiation, progression and metastasis (15). In tumors, neutrophils suppress $\mathrm{T}$ cell function, leading to tumor progression (16). Tumor-infiltrating lymphocytes, particularly those with Th1 polarization, are associated with improved prognosis (17).

Although neutrophils and lymphocytes are non-specific parameters that are affected by concurrent infection, inflammation, corticosteroids, chemotherapy and radiation therapy, baseline NLR is a promising, readily available and cost-effective biomarker that adds prognostic value to known clinical and laboratory prognostic factors $(3,8,18)$. In the present study, patients with an NLR $>4$ with metastatic disease that were referred to radiation oncology had a median survival of 4.1 months. Further investigation of NLR in specific subsets of patients, including those with specific tumor types, oligometastatic and widespread metastatic disease treated with radiation therapy, is warranted.

\section{Acknowledgements}

Not applicable.

\section{Funding}

Research support was provided by The Good Samaritan Hospital Medical Center Foundation (West Islip, NY, USA).

\section{Availability of data and materials}

The datasets used and/or analyzed during the current study are available from the corresponding author on reasonable request.

\section{Authors' contributions}

AW, AZ, DL, PK, M-KJ and JK made substantial contributions to conception and design, acquisition of data, and analysis and interpretation of data. AW, AZ, DL, PK, M-KJ and JK agree to be accountable for all aspects of the work in ensuring that questions related to the accuracy or integrity of any part of the work are appropriately investigated and resolved. AW, AZ, DL, PK and JK been involved in drafting the manuscript and revised it critically for important intellectual content. All the authors have read and approved the final version of this manuscript to be published.

\section{Ethics approval and consent to participate}

The Good Samaritan Hospital Medical Center IRB (no. 16-016) approved this minimal risk registry study with waiver of informed consent. There were no vulnerable populations.

\section{Patient consent for publication}

Not applicable.

\section{Competing interests}

The authors declare that they have no competing interests.

\section{References}

1. Hanahan D and Weinberg RA: Hallmarks of cancer: The next generation. Cell 144: 646-674, 2011.

2. Guthrie GJ, Charles KA, Roxburgh CS, Horgan PG, McMillan DC and Clarke SJ: The systemic inflammation-based neutrophil-lymphocyte ratio: Experience in patients with cancer. Crit Rev Oncol Hematol 88: 218-230, 2013.

3. Templeton AJ, McNamara MG, Šeruga B, Vera-Badillo FE, Aneja P, Ocaña A, Leibowitz-Amit R, Sonpavde G, Knox JJ, Tran B, et al: Prognostic role of neutrophil-to-lymphocyte ratio in solid tumors: A systematic review and meta-analysis. J Natl Cancer Inst 106: dju124, 2014.

4. Kao J, Gold KD, Zarrili G, Copel E, Silverman AJ, Ramsaran SS, Yens D and Ryu S: Clinical predictors of survival for patients with stage IV cancer referred to radiation oncology. PLoS One 10: e0124329, 2015.

5. Zucker A, Tsai CJ, Loscalzo J, Calves P and Kao J: The NEAT predictive model for survival in patients with advanced cancer. Cancer Res Treat 50: 1433-1443, 2018.

6. Chow E, Abdolell M, Panzarella T, Harris K, Bezjak A, Warde P and Tannock I: Predictive model for survival in patients with advanced cancer. J Clin Oncol 26: 5863-5869, 2008.

7. Krishnan MS, Epstein-Peterson Z, Chen YH, Tseng YD, Wright AA, Temel JS, Catalano P and Balboni TA: Predicting life expectancy in patients with metastatic cancer receiving palliative radiotherapy: The TEACHH model. Cancer 120: 134-141, 2014.

8. Kao J, Timmins J, Ozao-Choy J and Packer S: Effects of combined sunitinib and extracranial stereotactic radiotherapy on bone marrow hematopoiesis. Oncol Lett 12: 2139-2144, 2016.

9. Golden EB, Chhabra A, Chachoua A, Adams S, Donach M, Fenton-Kerimian M, Friedman K, Ponzo F, Babb JS, Goldberg J,et al: Local radiotherapy and granulocyte-macrophage colony-stimulating factor to generate abscopal responses in patients with metastatic solid tumours: A proof-of-principle trial. Lancet Oncol 16: 795-803, 2015.

10. Azab B, Camacho-Rivera M and Taioli E: Average values and racial differences of neutrophil lymphocyte ratio among a nationally representative sample of United States subjects. PLoS One 9: e112361, 2014.

11. Forget P, Khalifa C, Defour JP, Latinne D, Van Pel MC and De Kock $M$ : What is the normal value of the neutrophil-to-lymphocyte ratio? BMC Res Notes 10: 12, 2017.

12. Gomez DR, Blumenschein GR Jr, Lee JJ, Hernandez M, Ye R, Camidge DR, Doebele RC, Skoulidis F, Gaspar LE, Gibbons DL, et al: Local consolidative therapy versus maintenance therapy or observation for patients with oligometastatic non-small-cell lung cancer without progression after front-line systemic therapy: A multicentre, randomised, controlled, phase 2 study. Lancet Oncol 17: 1672-1682, 2016. 
13. Hong JC, Ayala-Peacock DN, Lee J, Blackstock AW, Okunieff P, Sung MW, Weichselbaum RR, Kao J, Urbanic JJ, Milano MT, et al Classification for long-term survival in oligometastatic patients treated with ablative radiotherapy: A multi-institutional pooled analysis. PLoS One 13: e0195149, 2018.

14. Kitamura T, Qian BZ and Pollard JW: Immune cell promotion of metastasis. Nat Rev Immunol 15: 73-86, 2015.

15. Coffelt SB, Wellenstein MD and de Visser KE: Neutrophils in cancer: Neutral no more. Nat Rev Cancer 16: 431-446, 2016.

16. Nicolas-Avila JA, Adrover JM and Hidalgo A: Neutrophils in homeostasis, immunity, and cancer. Immunity 46: 15-28, 2017.

17. Chen HM, Ma G, Gildener-Leapman N, Eisenstein $S$, Coakley BA, Ozao J, Mandeli J, Divino C, Schwartz M, Sung M, et al: Myeloid-derived suppressor cells as an immune parameter in patients with concurrent sunitinib and stereotactic body radiotherapy. Clin Cancer Res 21: 4073-4085, 2015.
18. Chowdhary M, Switchenko JM, Press RH, Jhaveri J, Buchwald ZS, Blumenfeld PA, Marwaha G, Diaz A, Wang D, Abrams RA, et al: Post-treatment neutrophil-to-lymphocyte ratio predicts for overall survival in brain metastases treated with stereotactic radiosurgery. J Neurooncol 139: 689-697, 2018. 